\title{
To speak with an accent: Effects of nasal distortion on stuttering under delayed auditory feedback
}

\author{
DONALD G. MacKAY, ${ }^{2,3}$ \\ UNIVERSITY OF CALIFORNIA, LOS ANGELES
}

The main question in this study was whether Ss stutter less under DAF when speaking with an accent such as a nasal twang. The hypothesis was verified: $A$ group of 21 Ss stuttered less under $D A F$ when speaking with a nasal accent than when speaking normally. This effect was shown to be due to: (1) The change in the acoustic output as a result of speaking with the accent. A passively produced distortion of the returning feedback (after Held, 1961), which was similar to the actively articulated nasal accent, also caused the Ss to stutter less than normally. (2) Paying less attention to the disruptive feedback. The Ss were shown to pay less attention to nasal feedback, probably because producing a nasal accent requires greater attention to articulation. These results were not due to a reduction in the intelligibility of the nasal feedback, which was just as intelligible as the S's normal voice, even in noise. The significance of these findings for general theories of $D A F$ and pathological stuttering were discussed.

Experimental delays in auditory feedback are known to cause stuttering in normal individuals (Lee, 1951). The present study of DAF investigated the following questions about stuttering under delayed auditory feedback (DAF): Do we stutter less under DAF when we speak with an unusual accent? Is the effect of speaking with an accent due simply to the change in the acoustic characteristics of the returning feedback? What is the relation between the intelligibility of the returning feedback and the extent of DAF interference? Can the effect of DAF on foreign speakers be attributed to their accents? Does DAF stuttering reflect an attempt to correct a distortion in feedback resulting from delaying the acoustic signal as postulated in servosystem theories? What is the relationship between DAF and pathological stuttering?

\section{EXPERIMENT 1:}

THE EFFECT OF ASSUMING AN UNUSUAL ACCENT

The first experiment was an attempt to demonstrate a parallel between normal and pathological stuttering. Bryngelson (1958) reported that when pathological stutterers put on or assume an unusual accent such as a Louisiana drawl, their stuttering is reduced. We were interested in whether normal Ss also stutter less under DAF when they speak with an accent.

\section{Table 1}

(a) The CSI (in seconds per syllable) for the Three Experimental Conditions: Nasal Voice, Normal Voice (reduced and maximal rate), where N Represents the Number of Ss. (b) The Probability of Stuttering (per syllable) for the Nasal and Normal Voice Conditions.

$$
\text { a b }
$$

Rate of Speech Probability of Stuttering (seconds per syllable) (per syllable)

Nasal Voice

$(\mathrm{N}=15)$

.304

.002

Normal Voice

(Reduced Rate)

$(\mathrm{N}=15)$

.314

.03

Normal Voice

(Maximal Rate)

$(\mathrm{N}=15)$

.250

.09
Subjects

The Ss were 15 students at UCLA, who were paid for their participation in the experiment. Their mean age was 26.

\section{Apparatus}

The apparatus for delaying feedback was an Echovox (Kay Electric Company) variable feedback device. The feedback delays were .175 and $.2 \mathrm{sec}$. For both delay times, $\mathrm{S}$ was wearing Permoflux (PDR 600) stereo earphones. A Monarch TM-18 microphone was adjusted to about 6 in. from his lips. The microphone was connected to a Knight (KN-724) Stereo Amplifier System which transmitted the S's speech to the earphones at an average sound pressure level of $95 \mathrm{~dB}$ in the .2-sec delay condition.

\section{Procedure}

For one condition the procedure was as follows: $S$ was presented with $10 \quad 15$-syllable sentences on index cards and instructed to read each sentence as fast as he could in his normal voice regardless of what happened to his speech under DAF.

The other condition was identical except that the Ss were instructed to read 10 sentences with a nasal accent. The $\mathrm{E}$ showed the $S$ how to nasalize speech (the result of contracting the velar muscles, Heffner, 1964). Each $S$ was given several sentences without DAF so that he could practice his nasal accent.

\section{Controls}

Feedback intensity. Since Ss in a pilot study tended to speak more softly than normally through their noses, the level of the nasal feedback was amplified to match that of the non-nasal feedback. Subsequent measurements in the main experiment showed that the nasal feedback was at least as loud as the non-nasal feedback.

The rate of speech control. Since speaking with a nasal accent tended to reduce the maximum rate of the Ss in the pilot study, another condition was added in which the speech rates in the nasal and non-nasal conditions were matched. To control for rate of speech, the Ss were instructed to speak at a normal rather than maximal rate for a set of 10 sentences.

The order of the three experimental conditions (nasal, non-nasal maximum rate, and non-nasal reduced rate) was counterbalanced across Ss. The three sets of sentences were shuffled, and the order of the delay conditions was randomly varied for each $S$.

\section{Results}

Rate of speech. The time to produce each sentence was determined with a standard-stop watch. The speech rate was estimated from a measure of syllable duration known as the correct syllable interval (CSI). The CSI is defined as $\mathrm{Tn} / \mathrm{S}$, where $\mathrm{Tn}$ is the time to correctly produce some proportion $n$ of the S syllables in the sentence (after Fairbanks, 1955).

The rates of speech for the three experimental conditions are compared in Table la. As might be expected, the mean CSI was smaller when the Ss were speaking at their maximum as compared to their normal rates. When speaking with a nasal accent the Ss spoke at about their normal rates (see Table 1), allowing valid comparison of DAF stuttering in the nasal and normal rate conditions 
$D A F$ stuttering. The probability of stuttering (per syllable) is shown in Table $1 b$ for the three experimental conditions. As can be seen there, the Ss stuttered less when speaking with a nasal accent than when speaking normally at either rate of speech. There was more stuttering in the reduced rate, normal voice condition than in the nasal condition, a difference significant at the .05 level using a two-tailed sign test with Ss as unit of analysis.

Another effect which can be seen in Table $1 \mathrm{~b}$ is that reducing the rate of speech tended to reduce DAF stuttering. The Ss stuttered less when speaking at their normal rates than at their maximal rates. This difference was significant at the .05 level using a two-tailed sign test. This finding agrees with other studies of speech rate under DAF (MacKay, in press; Kodman, 1961; and Beaumont \& Foss, 1957).

\section{Discussion}

The hypothesis was confirmed. Articulating a nasal accent reduced DAF stuttering. Subsequent experiments tested a number of alternative explanations of this result, namely that stuttering is reduced with a nasal accent because of (1) distortion of the returning feedback (the Distortion Hypothesis), (2) the pitch characteristics of the feedback (the Pitch Hypothesis), (3) a reduction in intelligibility of the returning feedback (the Intelligibility Hypothesis), and (4) paying more attention to articulation when speaking with an accent (the Attention Hypothesis).

\section{EXPERIMENT 2 :}

\section{THE DISTORTION HYPOTHESIS}

The second experiment tested whether the effect of a nasal accent is due to articulatory or to acoustic distortion factors.

Nasality in the acoustic signal can be accomplished in two ways. Hypernasality results when the velum opens the nose to the rest of the sypralaryngeal vocal tract while the nose is open to the atmosphere. Linguistic nasality as it occurs in the sound $\mathrm{ln} /$ is hypernasalized. When the Ss were asked to speak with a nasal accent in the above experiments, they were hypernasalizing phonemes that normally would not be nasalized.

But when the nose is blocked from the atmosphere, as when a speaker has a severe cold, hyponasality results. When a $\mathrm{S}$ speaks while holding his nose, he is hyponasalizing. However, as will be shown, acoustic properties of hyponasalizing and hypernasalizing are quite similar.

We reasoned that if the effect of a nasal accent in Experiment 1 were due to acoustic distortion factors, then a nasal distortion of the acoustic signal due to hyponasalization should effect DAF stuttering as much as an articulated nasal accent. The nasal distortion was created by having the Ss speak while holding their noses. This procedure is essentially equivalent to. Held's (1961) passive movement paradigm, with the nasal condition in the previous experiment corresponding to his active paradigm. Holding the nose created feedback basically similar to a nasal accent, without actual articulation of the accent by contraction of the velar muscles. If a nasal accent reduces DAF stuttering for acoustic reasons, the nasal distortion from holding the nose should have the same effect as articulating the nasal accent. Thus the Distortion Hypothesis predicts that stuttering under DAF will be reduced by speaking while holding the nose.

The Ss were eight students at UCLA, paid as before. Their average age was 21 . The procedures and materials were identical to those in Experiment 1 except that the instructions were as follows: "We wish to determine the effect of distorted feedback. For the following sentences we would like you to produce a nasal accent by holding your nose like this" ( $E$ demonstrates the procedure of holding the nose so the hand does not block the microphone).

The sentences were mounted on an adjustable stand so as to be easily read. As before, the $S$ was instructed to read the sentences at his maximum rate without worrying about errors.

\section{Results}

The rate of speech. The rate of speech for the nasal distortion and normal voice are shown in Table $2 \mathrm{a}$. As can be seen there, the Ss spoke at about the same rate with nasal distortion as when speaking at their normal rates of speech in their normal voices. This allowed valid comparison of DAF interference in these two conditions.

$D A F$ interference. The probability of stuttering is shown in Table $2 \mathrm{~b}$. Again, speaking at a slower rate reduced stuttering in the normal voice.

Nasal distortion reduced DAF interference, since the Ss stuttered less when speaking while holding their noses than when speaking normally at an equivalent rate of speech. Nasal distortion in Experiment 2 thus had a similar effect to nasal articulation in Experiment 1.

\section{Discussion}

The Distortion Hypothesis was confirmed, since nasal distortion in this experiment reduced DAF stuttering relative to the normal voice condition. A similar effect of feedback distortion was shown by Hull (1952) and Roehrig (1965) using frequency filters. The present findings both corroborate and extend their results for accent-like distortions.

However, stuttering was less in the nasal articulation condition than in the nasal distortion condition. Clearly, other factors are involved in voluntarily producing an accent. The next experiment tested whether the attention to articulation required in producing a nasal accent contributed to the difference between the actively and passively produced nasal accent.

\section{EXPERIMENT 3: \\ THE ATTENTION HYPOTHESIS}

The attention hypothesis assumes that speaking with an accent requires concentration on articulation so that the $S$ must ignore his auditory feedback. By paying less attention to feedback, interference under DAF is reduced according to the Attention Hypothesis.

In order to test this hypothesis, a technique for objectively determining attention to feedback was adopted (after MacKay, in press). In this "irrelevant voice procedure," the $S$ reads a sentence as before, but instead of hearing his own feedback, he hears another irrelevant voice. This irrelevant voice tended to reduce the normal speech rate (MacKay, in press). It was later shown that the greater the disruption from the irrelevant voice, the greater the interference under DAF, suggesting that the irrelevant voice procedure was a valid index of attention to feedback under DAF. ${ }^{4}$

Table 2

(a) The CSI for the Nasal Distortion and Normal Voice Conditions. (b) The Probability of Stuttering for the Nasal Distortion and Normal Voice Conditions.

\begin{tabular}{cc}
$\begin{array}{c}\text { Rate of Speech } \\
\text { (seconds per syllable) }\end{array}$ & $\begin{array}{c}b \\
\text { Probability of Stuttering }\end{array}$ \\
\hline
\end{tabular}

Nasal Distortion

(holding the nose)

$(\mathrm{N}=8)$

.200

.047

Normal Voice

(reduced rate)

$(\mathrm{N}=8)$

.071

Normal Voice

(Maximal Rate)

$(\mathrm{N}=8)$

.101

.228 
Table 3

The CSI for: (a) The Irrelevant Voice Condition and (b) The Zero Delay Condition for the Two Types of Nasal Speech, along with the Corresponding Distraction Ratios (i.e., a/b). See Text for Explanation.

\begin{tabular}{lcc} 
& \multicolumn{3}{c}{ Manner of Articulation } \\
\cline { 2 - 3 } & $\begin{array}{c}\text { Nasal Articulation } \\
(\mathrm{N}=15)\end{array}$ & $\begin{array}{c}\text { Nasal Distortion } \\
(\mathrm{N}=8)\end{array}$ \\
\hline $\begin{array}{l}\text { (a) Irrelevant } \\
\text { Voice Condition }\end{array}$ & .204 & .189 \\
$\begin{array}{l}\text { (b) Zero Delay } \\
\text { Condition }\end{array}$ & .262 & .185 \\
$\begin{array}{l}\text { (c) Distraction } \\
\text { Ratio (i.e., a/b) }\end{array}$ & .78 & 1.02 \\
\hline
\end{tabular}

Table 4

The CSI for: (a) The Irrelevant Voice Condition, and (b) The Zero Delay Condition for Nasal and Non-Nasal Speech with the Corresponding Distraction Ratios (i.e., a/b). See Text for Explanation.

\begin{tabular}{lcc}
\hline & $\begin{array}{c}\text { Nasal } \\
\text { (Articulation and Distortion) }\end{array}$ & $\begin{array}{c}\text { Normal } \\
\text { Articulation }\end{array}$ \\
\hline $\begin{array}{l}\text { (a) Irrelevant } \\
\text { Voice Condition }\end{array}$ & .197 & .182 \\
$\begin{array}{l}\text { (b) Zero Delay } \\
\text { Condition }\end{array}$ & .224 & .169 \\
$\begin{array}{l}\text { (c) Distraction } \\
\text { Ratio (i.e., a/b) }\end{array}$ & .88 & 1.07 \\
\hline
\end{tabular}

In the present experiment we used the irrelevant voice procedure to determine whether an $\mathrm{S}$ pays less attention to input when speaking with an accent. The instructions in the irrelevant voice condition were as follows: "This time you will hear an irrelevant voice while reading the sentence. As before, you are to read the sentence as fast as you can without paying attention to what you are hearing. There will be three manners of articulation: your normal voice, nasalization, and holding your nose (as before)."

The nature of the irrelevant voice varied with the manner of articulation. For example, when the $S$ was instructed to speak with a nasal accent, the irrelevant voice he heard was nasalized.

The irrelevant voice was, in fact, a recording of the S's own voice reading prose material either with his normal voice, a nasal accent, or while holding his nose. There was no playback of his voice as he read the test sentences, and as soon as he completed each sentence, the recording was turned off. Both the irrelevant recording and the zero delay feedback were amplified to about $95 \mathrm{~dB}$, the same average sound pressure level as in the .2-sec condition.

In the zero-delay condition the Ss were simply instructed to read the sentences as fast as they could either in their normal voices, with nasalization, or while holding their noses.

\section{Results}

The results for the nasal-articulation and nasal-distortion conditions are shown in Table 3 with their corresponding distraction ratios (the rate in the irrelevant-voice condition divided by the rate in the zero-delay condition). The distraction ratio in the nasal-articulation condition was much lower than in the nasal-distortion condition. This finding strengthens the suggestion that nasalization requires attention to articulation, which diverts attention from the auditory input. It also explains the lower probability of stuttering under DAF in the nasal articulation than in the nasal-distortion condition, which would be expected if less attention is being paid to the disruptive feedback with nasal articulation.

The irrelevant voice results for nasal and non-nasal speech are shown in Table 4 along with the corresponding distraction ratios. The distraction ratio for normal articulation was greater than it was for the nasal conditions. This difference was significant at the .05 level, using a two-tailed sign test with $S s$ as the unit of analysis.

\section{EXPERIMENT 4:}

\section{THE ACOUSTIC HYPOTHESIS}

Another possible factor in the difference in DAF stuttering for nasal and normal speech may be in the acoustic characteristics of nasal speech. We therefore compared the spectral properties of nasal and normal speech.

A male pilot $\mathrm{S}$ read the sentence, There once was a dog named Rover, into a spectrographic recorder (Kay Electric Company, Type B Sonogram). The $\mathrm{S}$ spoke at his maximum rate: (1) in his normal voice, (2) with a nasal accent, and (3) while holding his nose. For each condition he was instructed to repeat the phrase until his sound pressure level reached a predetermined level $(95 \mathrm{~dB})$ as indicated on the intensity meter of the Sonograph. This was necessary to make the acoustic analysis comparable to the DAF situation, where intensity was also equated. The three spectrograms are shown in Fig. 1 for the word dog from the test sentence (a broad-band intensity representation after Gleason, 1961). The dark-horizontal bands in Fig. 1 are formants, which represent a concentration of acoustic energy at various frequency levels. The fundamental pitch of the voice shows up in the concentration of the fine vertical bands in Fig. 1, and reflects the rate of vibration of the vocal cords in the larynx.

As can be seen in Fig. 1, the fundamental pitch of the voice was slightly higher for nasal than non-nasal speech (at most $60 \mathrm{~Hz}$ ). This is somewhat puzzling since there is no a priori reason why this should be the case. One possible explanation of the speaker's higher fundamental pitch is that he was phonating at a higher level of vocal effort in the nasal conditions. Nasalization introduces more damping in the supralaryngeal vocal tract. Consequently, to achieve the same sound pressure level as normal speech, the speaker would have to use more effort. This would result in a higher subglottal air pressure which would raise the pitch of his voice. We can only conclude that under these conditions, with this $S$, and with equalization of the sound pressure level of normal and nasal speech, the fundamental pitch is higher for the nasal accents.

Moreover, we still cannot conclude that pitch factors per se reduced the DAF stuttering in the nasal distortion case. Conceivably, distortion of the acoustic signal per se may have been the cause of this, rather than the particular distortion which occurred. Further research on the question is needed.
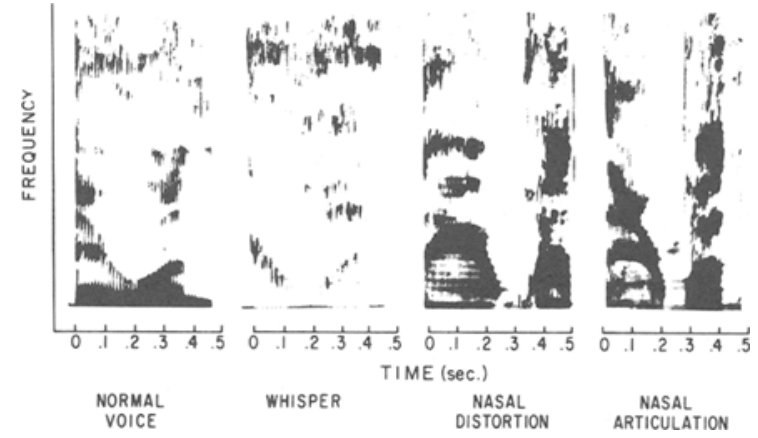

Fig. 1. A broad-band spectrogram of the word $d o g$ in the sentence There once was a dog named Rover for: (a) normal (voiced) speech, (b) whispered (unvoiced) speech (the spectrogram shows what may be some voicing at a very low fundamental in the whispered speech, a common effect during whispering), (c) nasally dist orted speech (holding the nose), and (d) nasally articulated speech. 
Table 5

The Intelligibility Scores (the probability of correct identification) for the Three Modes of Production: Nasalization, Nasal Distortion, and Normal Speech.

Intelligibility (the probability of correct identification per syllable).

\begin{tabular}{ccc} 
Normal Speech & Nasal Articulation & Nasal Distortion \\
\hline .23 & .25 & .22 \\
\hline
\end{tabular}

\section{Discussion}

Experiments 3 and 4, taken together, suggest that assuming an accent may reduce DAF stuttering for two reasons: (1) Acoustic distortion factors, i.e., nasal distortion of the acoustic signal, reduces DAF stuttering, either per se or because of spectral characteristics peculiar to nasal speech, and (2) Attention to articulation.

Two results favor the attention hypothesis: (1) the fact that the irrelevant voice was less distracting when the $S$ was speaking with a nasal accent, than in his normal voice, and (2) the fact that stuttering under DAF was reduced more by nasal articulation than by nasal distortion, where the Ss obviously would not have to pay attention to linguistic nasalization or non-nasalization.

This suggests that directing attention away from the returning feedback may reduce DAF stuttering. However, this does not imply that DAF stuttering is due to monitoring the returning feedback. Concentration on articulation may directly facilitate the control of speech, whether under DAF or not.

\section{EXPERIMENT 5:}

\section{THE INTELLIGIBILITY. HYPOTHESIS}

Several other investigators have reported that mechanical distortion of the acoustic signal reduces DAF stuttering. For example, Hull (1952) found that DAF disruption was reduced when he distorted their feedback with high and low pass frequency filters. However, since frequency distortion always alters the intelligibility of the speech signal (Roehrig, 1965), the reduced stuttering in these experiments could be attributed to reducing the intelligibility of the delayed feedback. We, therefore, wished to determine whether the effects of distortion in our previous experiments were due to changes in intelligibility. The plan was to present nasal and non-nasal sentences in noise, to determine whether nasal speech was less intelligible than non-nasal speech.

\section{Materials}

Six sentences from Experiment 1 were recorded on tape for each of the three manners of articulation: normal voice, nasal articulation, and holding the nose. Care was taken to ensure that the 18 sentences were equally loud (determined by the intensity meter of the Sonograph described above). A masking noise was added with a broad-band white-noise generator (General Radio Corporation Type 1390-13), in order to give less than $100 \%$ intelligibility. The order of the three types of articulation was counterbalanced for the 18 sentences.

Four graduate students at UCLA listened to the tape with the following instructions: "You will hear sentences in noise. After each sentence, repeat as much of it as you can, guessing if necessary." There was a $20-\mathrm{sec}$ pause after each sentence while $E$ recorded the number of syllables that $S$ identified correctly. The probability of correct identification (per syllable) is shown in Table 5. There were no significant differences in the intelligibility scores for any of the three types of production taken in pairs ( $t$ test, .50 level).

\section{Discussion}

Hull (1952) and Roehrig (1965) showed that acoustic distortion, combined with lowered intelligibility, reduced DAF stuttering. The present study shows that DAF distortion alone is sufficient to reduce DAF interference. Whether intelligibility has an effect on DAF interference independent of DAF distortion is as yet unknown. We can only conclude that distortion of feedback has an effect independent of changes in feedback intelligibility.

\section{CODA}

One of the questions in the introduction was whether DAF stuttering reflects an attempt to correct an apparent error resulting from delaying the acoustic feedback, a process postulated in servosystem theories of DAF. Two classes of servosystem theory can be discriminated; neither theory can explain the effect of DAF distortion without special assumptions.

One class of servosystem theories can be described as Output-Matching Models, since they hold that errors in speech under DAF reflect an attempt to restore the normal correspondence between speech output and feedback (Fairbanks, 1954). In this theory, the S stutters under DAF in order to make what he's saying match what he's hearing. By distorting the delayed feedback, correspondence between output and feedback would become more difficult to achieve, so that attempts to achieve this correspondence (by stuttering for example) should increase with DAF distortion. Since experiments on DAF distortion show the opposite, this class of servosystem theories must be either discarded or undergo special modification to account for the effects of feedback distortion.

The second class of servosystem theories can be described as Input-Correspondence Models, since they view DAF stuttering as an attempt to correct the "erroneous" feedback. Delayed auditory feedback creates a mismatch between actual and expected feedback according to this model. The $\mathrm{S}$ notes that his concurrent feedback under DAF doesn't match what he intended to say or expected to hear, and says it again. Since distortion of the returning feedback would further magnify the mismatch between output and expected feedback, DAF stuttering should have increased rather than decreased in the Roehrig (1965), Hull (1952), and the present studies. Since the opposite was found, this model must be seriously amended if it is to account for the effects of DAF distortion.

Treisman (1965) presents a similar view of DAF interference, postulating monitoring at an intelligibility or informational level. As well as the problems of servosystem theories discussed above, this theory also has the problem of explaining how feedback distortion can reduce DAF interference without changing the intelligibility of the returning signal (as shown in Experiment 5).

Another question in the introduction was whether the effect of DAF on foreign speakers can be attributed to their accents. Foreign accents bear a theoretical resemblance to the accent-like distortions in the present study. Stevens and Halle (1967) view the accent of a foreign speaker as an involuntary distortion of what he intends to say. That is, the foreigner may intend to speak his newly acquired language without an accent at some higher voluntary level of speech production, but old habits of pronunciation carry over from his native language, and inadvertently distort his output. However, MacKay has found ${ }^{4}$ that individuals stutter more under DAF rather than less in the language they speak with an accent, and in a series of experiments showed that DAF stuttering was a direct function of familiarity with language spoken under DAF. Consequently, if foreign accents are similar to feedback distortion their effect on DAF interference must be small and completely overshadowed by the effect of language familiarity.

To return at last to the question of a possible relationship between DAF and pathological stuttering, Bryngelson (1958) showed that assuming an accent also reduces pathological 
stuttering. However, without controls for speech rate and tests for attention to articulation, we cannot conclude that factors reducing DAF stuttering are the same as those reducing pathological stuttering when pathological stutterers put on an accent, although at present that would be the simplest assumption.

\section{A COMPARISON OF NASALIZATION AND WHISPERING}

Further research is needed to determine whether other manners of articulation have the same effect on DAF interference as nasalization. For example, whispering is similar to nasalization in several ways. Both are the result of contracting a set of muscles (usually the posterior crico artenoids of the larynx in the case of whispering although whispering can be effected in several ways) and both change the acoustic wave-form in a uniform manner (devoicing the speech signal in the case of whispering).

Despite these formal similarities, whispering and nasalization appear to have opposite effects on DAF interference. Lawrence (1964) found that Ss stuttered more when whispering than when speaking normally under DAF, with appropriate controls for intensity of the returning feedback.

In order to determine whether acoustic factors could account for the difference between whispering and nasalization, a sound spectrogram of whispered speech was determined as in Experiment 2. A broad-band frequency spectrum of the whispered word, $d o g$, in the context, "There once was a dog named Rover," is shown in Fig. lb.

Comparison of the same word in the normal voice of the same $S$ (shown in Fig. 1a) reveals the absence of a fundamental pitch in whispering. This is to be expected, of course, since the fundamental pitch (represented as the vertical lines in Fig. 1a) results from vocal fold vibration which does not occur in whispering (Heffner, 1964). Note also that the lower frequency formants of whispered speech appear almost nonexistent, whereas the high frequency formants appear more intense than the same formants in normal and nasal speech. This is an artifact reflecting the high frequency shaping and automatic gain-control electronics of the sound spectrograph rather than the acoustic quality of the whisper itself. Whispered noise excitation is essentially flat across the spectrum (to the first approximation). Relative to normal speech there is comparatively more energy at the high end of the spectrum. The sound spectrogram's high frequency preemphasis network emphasizes the high frequency intensity since for normal speech the intensity spectrum of the laryngeal source falls off at about $12 \mathrm{~dB}$ per octave (Fant, 1960). Similarly, the spectrograph has an automatic gain-control circuit which adjusts the signal level in terms of the overall signal in all frequency bands, reducing the "blackening" for the lower formants.

Thus, if an acoustic rather than articulatory interpretation of the difference between nasalization and whispering is sought, the lack of periodicity in the acoustic signal of whispering might be the place to search rather than the pitch spectrum. A spectral interpretation of the effect of whispering on DAF interference also appears inherently unreasonable, since higher frequencies are believed to contribute very little to DAF stuttering (Hull, 1952) and pathological stuttering (Ham \& Steer, 1967) relative to low frequencies. However, these studies of frequency filtering should be regarded as tentative, both because of the confounding of intelligibility with distortion and because of a lack of control for intensity of the filtered feedback. Control for intensity in these studies is especially important since the high frequency components in speech are known to be less intense than low ones (Rudmose et al, 1948), and intensity is a critical determinant of DAF interference (Black, 1951). Clearly, further research is needed to unambiguously determine the acoustic parameters of DAF interference, and to determine whether acoustic factors play a role in the difference between nasalization and whispering under DAF. It is interesting that whispering is thought to reduce rather than increase pathological stuttering (Eisenson, 1958). In this case, however, controls for intensity of the acoustic feedback of stutterers are imperative before this can be concluded with certainty.

Finally, the obtained difference between whispering and nasalization appears to limit the generality of other models of DAF interference. For example, Hochberg and Berko (1965) hold that under certain circumstances the $S$ may reject the returning feedback as not his own, thereby reducing DAF interference. This model is limited in explaining DAF distortion since, if it applies to nasal distortion, it cannot apply to whispering or vice versa.

Another (servosystem) model postulates that several different dimensions or features of the acoustic signal may be separately monitored. Voicing and nasality are examples of such features or dimensions. Thus, during whispering the voicing dimension assumes a constant value, implying that this dimension could be ignored in monitoring. With one less feature to monitor while whispering, DAF stuttering should decrease. However, the obtained increase in stuttering during whispering contradicts this model (V. Fromkin, personal communication, 1968).

\section{SUMMARY}

Speaking with a nasal accent was shown to reduce DAF stuttering without changing the intelligibility of the returning signal. This result was attributed to the concentration on articulation needed to produce the nasal accent. The possibility was discussed that similar factors reduce the stuttering of pathological stutterers when they put on an accent.

The relevance of feedback distortion to three servosystem theories of DAF was pointed out. One model viewed DAF stuttering as an attempt to make the delayed acoustic feedback and ongoing articulation correspond. Another model saw DAF stuttering as an attempt to correct ongoing articulation on the basis of the erroneous signal resulting from DAF. Another model claimed that DAF interference reflects a monitoring process at an intelligibility level. Without serious revision none of these models can explain the effects of feedback distortion on DAF interference.

\section{REFERENCES}

BLACK, J. W. The effect of delayed side-tone upon vocal rate and intensity. Journal of Speech \& Hearing Disorders, 1951, 16, 56-60.

BRYNGELSON, B. A study of speech difficulties of thirteen stutterers. In J. Eisenson (Ed.), Stuttering. New York: Harper and Row, 1958.

FAIRBANKS, G., \& GUTTMAN, N. Effects of delayed auditory feedback upon articulation. Journal of Speech \& Hearing Research, $1958,1,12-22$.

FAIRBANKS, G. Selective vocal effects of delayed auditory feedback. Journal of Speech \& Hearing Disorders, 1955, 20, 333-346.

FAIRBANKS, G. Systematic research in experimental phonetics: A theory of the speech mechanisms as a servo-system. Journal of Speech \& Hearing Disorders, 1954, 19, 133-139.

FANT, C. G. The acoustic theory of speech production. The Hague: Mouton, 1960.

FILLENBAUM, S., \& WIESEN, R. Contextual constraints and disruption in reading with delayed auditory feedback. Journal of the Acoustical Society of America, 1961, 33, 1800-1801.

GLEASON, H. A. Descriptive linguistics. New York: Holt, Rinehart, and Winston, 1961.

GUTTMAN, N. Experimental studies of the speech control system. Unpublished Ph.D. Thesis, University of Illinois, 1954.

HAM, R., \& STEER, M. D. Certain effects of alterations in auditory feedback. Folia Phoniatrica, 1967, 19, 53-62.

HELD, R. Exposure history as a factor in maintaining stability of perception and coordination. Journal of Nervous and Mental Disease, 1961, 132, 26-32. 
HEFFNER, R. M. S. General Phonetics. Madison: University of Wisconsin Press, 1964.

HOCHBERG, J., \& BERKO, M. J. "Phenomenal displacement" in delayed auditory feedback: I. Disparate inter-aural intensities. Psychonomic Science, 1965, 2, 389.

HULL, F. M. Experimental investigation of speech disturbance as a function of the frequency distortion of delayed auditory feedback. Unpublished Ph.D. Thesis, University of Illinois, 1952.

KODMAN, E. Controlled reading rate under delayed speech feedback. Journal of Auditory Research, 1961, 1, 186-193.

LAWRENCE, C. F. Delayed auditory feedback of whispered speech. Unpublished Ph.D. dissertation, Stanford University, 1965.

LEE, B. S. Some effects of side-tone delay. Journal of the Acoustical Society of America, 1950, 22, 639-640.

MacKAY, D. G. Metamorphosis of a critical interval: Age linked changes in the delay in auditory feedback producing maximal disruption with speech. Journal of the Acoustical Society of America, in press.

POTTER, R. K., KOPP, G. A., \& GREEN, H. C. Visible Speech. New York: Van Nostrand, 1947.

ROEHRIG, W. C. Addition of controlled distortion to delay of auditory feedback. Perceptual \& Motor Skills, 1965, 21, 407-413.

RUDMOSE, H. W., CLARK, K.C., CARLSON, F.D., EISENSTEIN, J.C., \& WALKER, R. A. Voice measurements with an audio spectrometer. Journal of the Acoustical Society of America, 1948, 20, 503-512.

STENGEL, E. Speech disorders and mental disorders. In A. V. S. Reuch and M. O'Connor (Eds.), Disorders of language. London: Churchill Ltd., 1964.
STETSON, R. H. Motor phonetics: A study of speech movements in action. Amsterdam: Holland Publishing, 1951.

STEVENS, K. N., \& HALLE, M. Remarks on analysis by synthesis and distinctive features. In W. Wathan-Dunn (Ed.), Proceedings of the symposium on models for the perception of speech and visual form. Cambridge: M.I.T. Press, 1967.

TREISMAN, A. M. Reading rate, word information and auditory monitoring of speech. Nature, 1965, 205, 1297-1300.

\section{NOTES}

1. This work was supported by a grant from the University of California, Los Angeles (Number 2428), and by funds of the Department of Psychology, UCLA, provided by Dr. F. N. Jones.

2. Address: Department of Psychology, University of California, Los Angeles, California 90024.

3. The author wishes to thank Drs. Edward Carterette, Donald Lindsley, George Mount, and Donald Walter for generously lending various pieces of equipment for the present studies; and $\mathbf{K}$. Achevski and J. Nosaki for help in setting up and running the experiment, and in analyzing the data.

4. MacKay, D. G. Of age and experience: Factors in the control of speech under delayed auditory feedback, unpublished study.

(Accepted for publication October 14, 1968.) 